\title{
WAVE SPLITTING FOR FIRST-ORDER SYSTEMS OF EQUATIONS
}

\author{
G. CAVIGLIA and A. MORRO
}

Received 23 October 2001

Systems of first-order partial differential equations are considered and the possible decomposition of the solutions in forward and backward propagating is investigated. After a review of a customary procedure in the space-time domain (wave splitting), attention is addressed to systems in the Fourier-transform domain, thus considering frequencydependent functions of the space variable. The characterization is given for the direction of propagation and applications are developed to some cases of physical interest.

2000 Mathematics Subject Classification: 74J05, 35F10, 35Q72.

1. Introduction. Wave propagation in inhomogeneous media and reflection and transmission processes are associated with various problems of mathematical interest. The governing equations are generally partial differential equations of various orders with variable coefficients. A basic question is whether and how the solution to the governing equations can be decomposed into forward- and backward-propagating waves. This question is of great interest in that the result of a scattering, or reflectiontransmission, process is required to consist of outgoing waves. According to the literature, the decomposition is often performed in the space-time domain through a wave-splitting technique [5]. This technique works in homogeneous materials. Otherwise, the technique generates basis functions which though are not solutions to the governing equations. Also, even for homogeneous materials, the wave splitting is performed by carrying over to dispersive media (e.g., via convolutions) the propagation property of the principal, nondispersive part (see Section 2).

The governing equations are, for example, in the form (cf. [1])

$$
\partial_{z}^{2} u-c^{-2}(z) \partial_{t}^{2} u-a(z) \partial_{t} u-b(z) \partial_{z} u-d(z) u=0,
$$

in the unknown function $u(z, t), z \in \mathbb{R}, t \in \mathbb{R}^{+}$, where $c>0$ and $a \geq 0$. The dependence of the coefficients $a, b, c, d$ on the space variable $z$ means that we allow the material system to be inhomogeneous. Otherwise, the model equations can be given the form of a first-order system

$$
\partial_{z} w=C \partial_{t} w
$$

where $w$ is an $n$-tuple of unknown functions of $z$ and $t$. The matrix $C$ may have values in $\mathbb{C}^{n \times n}$, with numerical entries possibly dependent on $z$, or may contain operators such that $n$-tuples of functions $\partial_{t} w$ are mapped into $n$-tuples of functions $\partial_{z} w$. 
To our mind, a more satisfactory approach is possible only within the Fouriertransform domain. In such a case, the solution to the Cauchy problem for monochromatic components is determined explicitly. Because of the inhomogeneity of the material, the solution is shown to result from a mixture of the components (mode conversion) thus making the wavesplitting in the space-time scarcely significant. For each monochromatic component, we characterize the forward or backward propagation through the phase function. Next, we apply the characterization to some cases of physical interest. They show that the direction of propagation may change with the frequency, which proves that a claimed direction of a solution in the space-time domain may cease to be true even in homogeneous materials.

2. Wave splitting in time domain. Let $n$ be an even integer, $w: \mathbb{R} \rightarrow \mathbb{R}^{n}, A_{0} \in \mathbb{R}^{n \times n}$, $A^{\prime}: \mathbb{R}^{+} \rightarrow \mathbb{R}^{n \times n}$. We assume that the pertinent equations can be written in the form

$$
\partial_{z} w=\left(A_{0}+A^{\prime} *\right) \partial_{t} w=: A \mathcal{A}
$$

This means that we are considering problems in the space-time variables $z, t$.

The wave splitting is accomplished by determining a new $n$-tuple of unknown functions such that the corresponding system involves a diagonal matrix. Let $B_{0} \in \mathbb{R}^{n \times n}$, $B^{\prime}: \mathbb{R}^{+} \rightarrow \mathbb{R}^{n \times n}$ and consider $w^{f}, w^{b} \in \mathbb{R}^{n / 2}$ such that

$$
\left[\begin{array}{l}
w^{f} \\
w^{b}
\end{array}\right]=\left(B_{0}+B^{\prime} *\right) w=: \Re w
$$

If $\partial_{t} w^{f}$ and $\partial_{t} w^{b}$ are continuous, then

$$
\partial_{t}\left(\mathscr{B}\left[\begin{array}{l}
w^{f} \\
w^{b}
\end{array}\right]\right)=\mathscr{B}_{t}\left[\begin{array}{l}
w^{f} \\
w^{b}
\end{array}\right],
$$

that is, the convolution $\mathscr{B}$ and the derivative $\partial_{t}$ commute. Now, observe that

$$
\partial_{z}\left[\begin{array}{l}
w^{f} \\
w^{b}
\end{array}\right]=\mathscr{B}_{z} w=\mathscr{B} \mathscr{A}^{-1}\left[\begin{array}{l}
w^{f} \\
w^{b}
\end{array}\right] .
$$

It is asserted that $w^{f}$ and $w^{b}$ are regarded as forward- and backward-propagating if $\mathscr{B} A \mathscr{B}^{-1}$ is diagonal and the corresponding entry is appropriate. Upon solving suitable Volterra integral equations, the diagonal terms are determined, and, for example, the equation for $w^{f}$ takes the form (see [5, page 120])

$$
\left[\partial_{z}+c^{-1}(1+\xi *) \partial_{t}\right] w^{f}=0
$$

While it is obvious that such $w^{f}$ is a forward-propagating wave if $c$ is constant and $\xi \simeq 0$, we show later that an appropriate restriction on $\xi$ is required so that the forward-propagating character is maintained. To gain this end, we find it convenient, if not imperative, to argue in the Fourier-transform domain. 
3. First-order system in the Fourier-transform domain. Also, for specific applications, it is worth considering the equations of a dissipative material such as a viscoelastic body. We consider a one-dimensional viscoelastic solid along the $z$-axis. Let $u$ be a transverse component of the displacement. The equation of motion takes the form

$$
\rho \partial_{t}^{2} u=\partial_{z} \tau
$$

where $\rho$ is the mass density and $\tau$ is the traction component, namely,

$$
\tau=\mu_{0} \partial_{z} u+\int_{0}^{\infty} \mu^{\prime}(\xi) \partial_{z} u(t-\xi) d \xi .
$$

Here, $\mu_{0}$ is the instantaneous shear modulus and $\mu^{\prime}$, on $\mathbb{R}^{+}$, is the kernel characterizing the fading memory of the material. It is understood that $\rho, \mu_{0}$, and $\mu^{\prime}(\cdot)$ may depend on $z$, which means that the body is allowed to be inhomogeneous. It is convenient to extend $\mu^{\prime}(z, \cdot)$ to $\mathbb{R}$ by letting $\mu^{\prime}(z, \xi)=0$ as $\xi<0$.

In terms of $u$ and $\tau$, we write the equations as

$$
\partial_{z} \tau=\rho \partial_{t}^{2} u, \quad \tau=\left(\mu_{0}+\mu^{\prime} *\right) \partial_{z} u .
$$

Denote by the subscript $F$ the Fourier transform

$$
u_{F}(z, \omega)=\int_{-\infty}^{\infty} u(z, t) \exp (-i \omega t) d t .
$$

Application of the Fourier transform yields

$$
\partial_{z} \boldsymbol{T}_{F}=-\rho \omega^{2} u_{F}, \quad \boldsymbol{\tau}_{F}=\left(\mu_{0}+\mu_{F}^{\prime}\right) \partial_{z} u_{F}
$$

Consequently, the pair $w=[u, \tau]$ satisfies the first-order system of ordinary equations

$$
\partial_{z} w_{F}=A w_{F}
$$

where

$$
A=\left[\begin{array}{cc}
0 & \frac{1}{\mu_{0}+\mu_{F}^{\prime}} \\
-\rho \omega^{2} & 0
\end{array}\right] .
$$

As a second case, we consider (1.1). Apply the Fourier transformation to obtain

$$
\partial_{z}^{2} u_{F}+c^{-2} \omega^{2} u_{F}-i \omega a u_{F}-b \partial_{z} u_{F}-d u_{F}=0 .
$$

The pair $w=\left[u, \partial_{z} u\right]$ then satisfies system (3.6) where

$$
A=\left[\begin{array}{cc}
0 & 1 \\
d+i \omega a-\omega^{2} c^{-2} & b
\end{array}\right] .
$$

Incidentally, upon Fourier transformation system, (1.2) takes the form (3.6) with $A=i \omega C$ if $C \in \mathbb{C}^{n \times n}$. Otherwise, if convolutions or time derivatives are involved in $C$, then $A$ is determined by the Fourier transform properties. Anyway, we allow $A$ in (3.6) to depend on $z$ and be parametrized by the angular frequency $\omega$. 
4. Wave propagation in the Fourier-transform domain. Assume that the problem under consideration takes the form (3.6) and that the matrix $A$ is simple; namely, it has $n$ linearly independent eigenvectors. Let $\lambda_{1}, \ldots, \lambda_{n}$ be the eigenvalues, and $p_{1}, \ldots, p_{n} \in$ $\mathbb{C}^{n}$ the associated eigenvectors. For brevity, we omit specifying that each quantity is parameterized by $\omega$. Moreover, let $P$ be the matrix whose columns are the eigenvectors of $A$, that is,

$$
P=\left[p_{1}, \ldots, p_{n}\right]
$$

Also, let

$$
s=P^{-1} w_{F}
$$

Substitution of $w_{F}=P s$ in (3.6) provides

$$
\partial_{z} s=\Lambda s+Q s
$$

where

$$
\Lambda=P^{-1} A P=\operatorname{diag}\left[\lambda_{1}, \ldots, \lambda_{n}\right], \quad Q=-P^{-1} \partial_{z} P .
$$

If the material is homogeneous, then $\partial_{z} P=0$, and hence system (4.3) takes the diagonal form

$$
\partial_{z} s=\Lambda s
$$

where $\Lambda$ is independent of $z$. If, instead, the material is inhomogeneous $\left(\partial_{z} P \neq 0\right)$, then in general, $Q$ is a nondiagonal matrix and equations in (4.3) are not decoupled.

Assume that $A$ is independent of $z, \partial_{z} P=0$, and hence (4.5) holds. If $s$ is known at a value of $z$, say $z=0$, we have

$$
s(z)=\exp [\Lambda z] s(0)
$$

If, instead, (4.3) holds with a nonzero $Q$, then the application of $\exp \left[-\int_{0}^{z} \Lambda(\zeta) d \zeta\right]$ to (4.3) and the use of

$$
v=\exp \left[-\int_{0}^{z} \Lambda(\zeta) d \zeta\right] s
$$

give

$$
\partial_{z} v=F v, \quad v(0)=s(0)=P^{-1}(0) w(0)
$$

where

$$
F(z)=\exp \left[-\int_{0}^{z} \Lambda(\zeta) d \zeta\right] Q(z) \exp \left[\int_{0}^{z} \Lambda(\zeta) d \zeta\right] .
$$

To solve (4.8), we consider the propagator matrix $\Omega(z)$ such that

$$
v(z)=\Omega(z) v(0)
$$


It follows that

$$
\partial_{z} \Omega=F \Omega, \quad \Omega(0)=I
$$

where $I$ is the $n \times n$ identity matrix. Hence, we have

$$
\Omega(z)-I=\int_{0}^{z} F(\zeta) \Omega(\zeta) d \zeta .
$$

Upon the assumption that $F$ is bounded, the solution $\Omega$ exists, is unique in $L^{2}(\mathbb{R})$, and is given by the Neumann series (cf. [2,6])

$$
\Omega(z)=I+\sum_{m=1}^{\infty} \int_{0}^{z} F_{m}(z, \zeta) d \zeta
$$

where the sequence of functions $\left\{F_{m}\right\}$ is defined by

$$
F_{1}(z, \zeta)=F(\zeta), \quad F_{n+1}(z, \eta)=\int_{\eta}^{z} F_{n}(z, v) d v F(\eta)
$$

Consequently, we find that $s(z)=\exp \left[\int_{0}^{z} \Lambda(\zeta) d \zeta\right] v(z)$ is given by

$$
s(z)=\exp \left[\int_{0}^{z} \Lambda(\zeta) d \zeta\right] s(0)+\exp \left[\int_{0}^{z} \Lambda(\zeta) d \zeta\right] \mathscr{F}(z, \omega) s(0),
$$

where

$$
\mathscr{F}(z)=\sum_{m=1}^{\infty} \int_{0}^{z} F_{m}(z, \zeta) d \zeta .
$$

This in turn allows the original $n$-tuple $w_{F}$ to be written as

$$
\begin{aligned}
w_{F}(z)= & P(z) \exp \left[\int_{0}^{z} \Lambda(\zeta) d \zeta\right] P^{-1}(0) w_{F}(0) \\
& +P(z) \exp \left[\int_{0}^{z} \Lambda(\zeta) d \zeta\right] \mathscr{F}(z) P^{-1}(0) w_{F}(0)
\end{aligned}
$$

We now go back to the space-time domain through the inverse Fourier transform in the form

$$
\begin{aligned}
w(z, t)= & \frac{1}{2 \pi} \int_{-\infty}^{\infty} P(z, \omega) \exp \left[\int_{0}^{z} \Lambda(\zeta, \omega) d \zeta+i \omega t I\right] P^{-1}(0, \omega) w_{F}(0, \omega) d \omega \\
& +\frac{1}{2 \pi} \int_{-\infty}^{\infty} P(z, \omega) \exp \left[\int_{0}^{z} \Lambda(\zeta, \omega) d \zeta+i \omega t\right] \mathscr{F}(z, \omega) P^{-1}(0, \omega) w_{F}(0, \omega) d \omega
\end{aligned}
$$

where the dependences on $\omega$ are denoted explicitly.

Result (4.18) provides the solution $w(z, t)$ to system (3.6) with initial data on $w(0, t)$. 
5. Wave splitting in the Fourier-transform domain. Both integrals in (4.18) involve a superposition through the matrices $P(z, \omega), P^{-1}(0, \omega)$, and $\mathscr{F}(z, \omega)$ of functions of the form

$$
f_{\omega}(z, t):=\exp \left[\alpha_{\omega}(z)\right] \exp \left\{i\left[\phi_{\omega}(z)+\omega t\right]\right\}
$$

Now, if $g$ represents a forward-propagating wave in the form $g(z-v t)$, where $v>0$, then

$$
\partial_{t} g \partial_{z} g<0
$$

For a backward-propagating wave $h(z+v t)$, we have $\partial_{t} h \partial_{z} h>0$. Hence, we consider the real and imaginary parts of $f_{\omega}$, namely,

$$
\begin{aligned}
\xi_{\omega}(z, t) & :=\exp \left[\alpha_{\omega}(z)\right] \cos \left[\phi_{\omega}(z)+\omega t\right], \\
\eta_{\omega} & :=\exp \left[\alpha_{\omega}(z)\right] \sin \left[\phi_{\omega}(z)+\omega t\right],
\end{aligned}
$$

and evaluate the products $\partial_{t} \xi_{\omega} \partial_{z} \xi_{\omega}$ and $\partial_{t} \eta_{\omega} \partial_{z} \eta_{\omega}$. We have

$$
\begin{aligned}
\partial_{t} \xi_{\omega} \partial_{z} \xi_{\omega}= & \omega \partial_{z} \phi_{\omega}(z) \exp \left[2 \alpha_{\omega}(z)\right] \sin ^{2}\left[\phi_{\omega}(z)+\omega t\right] \\
& +\omega \partial_{z} \alpha_{\omega}(z) \exp \left[2 \alpha_{\omega}(z)\right] \sin \left[\phi_{\omega}(z)+\omega t\right] \cos \left[\phi_{\omega}(z)+\omega t\right], \\
\partial_{t} \eta_{\omega} \partial_{z} \eta_{\omega}= & \omega \partial_{z} \phi_{\omega}(z) \exp \left[2 \alpha_{\omega}(z)\right] \cos ^{2}\left[\phi_{\omega}(z)+\omega t\right] \\
& -\omega \partial_{z} \alpha_{\omega}(z) \exp \left[2 \alpha_{\omega}(z)\right] \sin \left[\phi_{\omega}(z)+\omega t\right] \cos \left[\phi_{\omega}(z)+\omega t\right]
\end{aligned}
$$

In both cases, the term involving $\partial_{z} \alpha_{\omega} \sin \left[\phi_{\omega}(z)+\omega t\right] \cos \left[\phi_{\omega}(z)+\omega t\right]$ is not definite. However, it is periodic in time with period $T=\pi /|\omega|$ and, for any time $t_{0} \in \mathbb{R}$,

$$
\begin{aligned}
\int_{t_{0}}^{t_{0}+T} \omega \partial_{z} & \alpha_{\omega}(z) \exp \left[2 \alpha_{\omega}(z)\right] \sin \left[\phi_{\omega}(z)+\omega t\right] \cos \left[\phi_{\omega}(z)+\omega t\right] d t \\
= & \frac{1}{2} \omega \partial_{z} \alpha_{\omega}(z) \exp \left[2 \alpha_{\omega}(z)\right] \int_{t_{0}}^{t_{0}+T} \sin \left[2 \phi_{\omega}(z)+2 \omega t\right] d t=0 .
\end{aligned}
$$

Now,

$$
\int_{t_{0}}^{t_{0}+T} \sin ^{2}\left[\phi_{\omega}(z)+\omega t\right] d t=\frac{\pi}{2|\omega|}
$$

and the same result holds for $\cos ^{2}\left[\phi_{\omega}(z)+\omega t\right]$. Hence, we have

$$
\int_{t_{0}}^{t_{0}+T} \partial_{t} \xi_{\omega} \partial_{z} \xi_{\omega} d t=\frac{\pi}{2}(\operatorname{sgn} \omega) \partial_{z} \phi_{\omega}(z) \exp \left[2 \alpha_{\omega}(z)\right]
$$

and the same result holds for $\partial_{t} \eta_{\omega} \partial_{z} \eta_{\omega}$. Accordingly, propagation in one direction is characterized as follows. 
DEFINITION 5.1. The function $f_{\omega}=\xi_{\omega}+i \eta_{\omega}$ of (5.1) represents a forward- (backward-) propagating wave if

$$
\omega \partial_{z} \phi_{\omega}(z)<0 \quad(>0)
$$

It is natural to apply Definition 5.1 to plane (monochromatic) waves where

$$
\alpha_{\omega}=0, \quad \phi_{\omega}(z)= \pm \frac{\omega z}{c}, \quad c>0 .
$$

We have

$$
\omega \partial_{z} \phi_{\omega}(z)= \pm \frac{\omega^{2}}{c}
$$

and hence $\phi_{\omega}(z)=\omega z / c$ corresponds to a backward-propagating wave and $\phi_{\omega}(z)=$ $-\omega z / c$ corresponds to a forward-propagating wave.

Now that the definition of wave propagating in one direction is available, we can investigate whether a pertinent field may be represented in terms of forward- and backward-propagating waves.

6. Applications. We now look for the wave splitting in the Fourier-transform domain relative to particular systems of equations.

6.1. Dispersive media. Apply the Fourier transform to (2.5) to have

$$
\partial_{z} w_{F}^{f}=-c^{-1}\left(1+\xi_{F}\right) i \omega w_{F}^{f} .
$$

Hence, the eigenvalue

$$
\lambda=-c^{-1}\left(1+\xi_{F}\right) i \omega
$$

implies that

$$
\phi_{\omega}(z)=-\omega \int_{0}^{z} c^{-1}(\zeta)\left(1+\Re \xi_{F}\right)(\zeta) d \zeta, \quad \alpha_{\omega}(z)=\omega \int_{0}^{z} c^{-1}(\zeta) \mathfrak{J} \xi_{F}(\zeta) d \zeta .
$$

Hence, we have

$$
\omega \partial_{z} \phi_{\omega}=-\omega^{2} c^{-1}(z)\left(1+\Re \xi_{F}\right)(z) .
$$

The wave is forward propagating if

$$
\mathfrak{R} \xi_{F}>-1
$$

If, instead, $\mathfrak{R} \xi_{F}<-1$, then the wave is backward propagating. Incidentally,

$$
\partial_{z} \alpha_{\omega}=c^{-1}(z) \mathfrak{J} \xi_{F}(z) .
$$

Consequently, we can have a forward-propagating $\left(\mathfrak{R} \xi_{F}>-1\right)$ wave while the sign of $\partial_{z} \alpha_{\omega}$ is unrestricted. This shows that the forward-propagating character is related to the condition $\Re \xi_{F}>-1$ of the kernel $\xi$ and is not automatically induced by the principal part $\partial_{z}+c^{-1} \partial_{t}$ of the operator in (2.5). 
6.2. Dissipative wave equation. For the sake of simplicity, we consider (1.1) with $d, b=0$, and hence (3.6) holds with

$$
w=\left[\begin{array}{c}
u \\
\partial_{z} u
\end{array}\right], \quad A=\left[\begin{array}{cc}
0 & 1 \\
i \omega a-\omega^{2} c^{-2} & 0
\end{array}\right] .
$$

The eigenvalues $\lambda$ of $A$ satisfy

$$
\lambda^{2}=-\omega^{2} c^{-2}+i \omega a
$$

Since $a>0$, the number $i \omega a-\omega^{2} c^{-2}$ is in the second quadrant. The eigenvalues $\lambda_{1}$ and $\lambda_{2}$ are then given by

$$
\lambda_{1}=\frac{|\omega|}{c \sqrt{2}}\left(\sqrt{\sqrt{1+\left(a c^{2} / \omega\right)^{2}}-1}+i \operatorname{sgn} \omega \sqrt{\sqrt{1+\left(a c^{2} / \omega\right)^{2}}+1}\right), \quad \lambda_{2}=-\lambda_{1} .
$$

Hence, we have

$$
\begin{aligned}
& \alpha_{\omega}(z)=\frac{|\omega|}{\sqrt{2}} \int_{0}^{z} c^{-1}(\zeta) \sqrt{\sqrt{1+\left(a c^{2} / \omega\right)^{2}(\zeta)}-1} d \zeta, \\
& \phi_{\omega}(\zeta)=\frac{|\omega|}{\sqrt{2}} \int_{0}^{z} c^{-1}(\zeta) \sqrt{\sqrt{1+\left(a c^{2} / \omega\right)^{2}(\zeta)}+1} d \zeta,
\end{aligned}
$$

as $\lambda=\lambda_{1}$ (and the opposite as $\lambda=\lambda_{2}$ ). Since

$$
\omega \partial_{z} \phi_{\omega}=\frac{\omega^{2}}{\sqrt{2}} c^{-1}(z) \sqrt{\sqrt{1+\left(a c^{2} / \omega\right)^{2}(z)}-1}>0,
$$

it follows that

$$
\exp \left(\int_{0}^{z} \lambda_{1}(\zeta) d \zeta+i \omega t\right)
$$

is a backward-propagating wave. This is consistent with the fact that

$$
\partial_{z} \alpha_{\omega}(z)=\frac{|\omega|}{\sqrt{2}} c^{-1}(z) \sqrt{\sqrt{1+\left(a c^{2} / \omega\right)^{2}(z)}-1}>0,
$$

and hence the amplitude decreases as $z$ decreases as is natural of a backward-propagating wave. Of course, if $a=0$, then

$$
\omega \partial_{z} \phi_{\omega}<0, \quad \partial_{z} \alpha_{\omega}=0
$$

and hence $\exp \left(\int_{0}^{z} \lambda_{1}(\zeta) d \zeta+i \omega t\right)$ propagates with a constant amplitude. In fact, the occurrence of

$$
P=\left[\begin{array}{cc}
1 & 1 \\
\lambda_{1} & -\lambda_{1}
\end{array}\right]
$$


and the dependence of $c$ on $z$ make $w$ a $z$-dependent combination of the two elementary waves

$$
\exp \left(\int_{0}^{z} \lambda_{1}(\zeta) d \zeta+i \omega t\right), \quad \exp \left(-\int_{0}^{z} \lambda_{1}(\zeta) d \zeta+i \omega t\right) .
$$

6.3. Shear waves in viscoelastic solids. System (3.6) holds with $A$ as given by (3.7). Since $\mu^{\prime}=0$ on $\mathbb{R}^{-}$, we have

$$
\mu_{F}^{\prime}(\omega)=\int_{0}^{\infty} \mu^{\prime}(\xi) \exp (-i \omega \xi) d \xi=\mu_{c}^{\prime}(\omega)-i \mu_{s}^{\prime}(\omega),
$$

where $\mu_{c}^{\prime}$ and $\mu_{s}^{\prime}$ are the half-range cosine and sine Fourier transforms of $\mu^{\prime}$. Of course $\mu_{s}^{\prime}(\omega)$ is an odd function of $\omega$. Also, by thermodynamics, we know that (cf. [3])

$$
\omega \mu_{s}^{\prime}(\omega) \leq 0, \quad \forall \omega \in \mathbb{R} .
$$

In addition, it is reasonable to assume that $\mu_{0}+\mu_{c}^{\prime}(\omega)>0$ for every $\omega \in \mathbb{R}$.

The eigenvalues $\lambda$ satisfy

$$
\lambda^{2}=-\frac{\rho \omega^{2}}{\mu_{0}+\mu_{c}^{\prime}-i \mu_{s}^{\prime}}
$$

or

$$
\lambda^{2}=\frac{\rho \omega^{2}}{\left[\left(\mu_{0}+\mu_{c}^{\prime}\right)^{2}+\left(\mu_{s}^{\prime}\right)^{2}\right]^{1 / 2}} \exp (i \theta)
$$

where

$$
\theta=\pi-\psi \operatorname{sgn} \omega, \quad \tan \psi=\frac{\left|\mu_{s}^{\prime}\right|}{\mu_{0}+\mu_{c}^{\prime}}
$$

Hence, we have

$$
\lambda_{1}=\sqrt{\frac{\rho \omega^{2}}{\left[\left(\mu_{0}+\mu_{c}^{\prime}\right)^{2}+\left(\mu_{s}^{\prime}\right)^{2}\right]^{1 / 2}}} \exp (i \theta / 2), \quad \lambda_{2}=-\lambda_{1},
$$

where

$$
\exp (i \theta / 2)=\operatorname{sgn} \omega \sqrt{\frac{\sqrt{1+\tan ^{2} \psi}-1}{2 \sqrt{1+\tan ^{2} \psi}}}+i \sqrt{\frac{\sqrt{1+\tan ^{2} \psi}-1}{2 \sqrt{1+\tan ^{2} \psi}}} .
$$

Consequently,

$$
\exp \left(\int_{0}^{z} \lambda_{1}(\zeta) d \zeta+i \omega t\right)=\exp \left(\alpha_{\omega}(z)\right) \exp \left[i\left(\phi_{\omega}(z)+\omega t\right)\right]
$$


where

$$
\begin{aligned}
& \alpha_{\omega}(z)=\omega \int_{0}^{z} \sqrt{\frac{\rho}{\mu_{0}+\mu_{c}^{\prime}} \frac{\sqrt{1+\tan ^{2} \psi}-1}{2\left(1+\tan ^{2} \psi\right)}}(\zeta) d \zeta, \\
& \phi_{\omega}(z)=|\omega| \int_{0}^{z} \sqrt{\frac{\rho}{\mu_{0}+\mu_{c}^{\prime}} \frac{\sqrt{1+\tan ^{2} \psi}+1}{2\left(1+\tan ^{2} \psi\right)}}(\zeta) d \zeta .
\end{aligned}
$$

Now,

$$
\omega \partial_{z} \phi_{\omega}=\omega|\omega| \sqrt{\frac{\sqrt{1+\tan ^{2} \psi}-1}{2 \sqrt{1+\tan ^{2} \psi}}}
$$

and then

$$
\omega \partial_{z} \phi_{\omega}>0 \text { as } \omega>0, \quad \omega \partial_{z} \phi_{\omega}<0 \text { as } \omega<0 .
$$

Accordingly, as $\omega>0$, the wave associated with $\lambda_{1}$ is backward propagating and, as we expect it to be, $\exp \alpha_{\omega}(z)$ increases as $z$ increases. If, instead, $\omega<0$, then the wave is forward propagating and $\exp \alpha_{\omega}(z)$ decreases as $z$ increases. The opposite behaviour occurs with the wave associated with $\lambda_{2}$.

7. Comments. The dissipative wave equation associated with (6.7) allows us to establish a connection with the wave splitting technique (cf. [4]). Consider [4, (7)] with $\kappa=0$ which coincides with (1.1) if $b, d=0$, in the time domain, or (6.7) in the frequency domain. Via the operator $K$ such that

$$
K^{-1}=\left(-c^{-2} \partial_{t}^{2}+a \partial_{t}\right) K,
$$

the functions

$$
u^{ \pm}=\frac{1}{2}\left(u \mp K \partial_{z} u\right)
$$

are considered. It is not claimed that $u^{+}$or $u^{-}$are forward- or backward-propagating waves, but it is taken to be so if the medium is homogeneous as $z<0$ or $z>L$. In such a case, the first-order system for $u^{+}, u^{-}$decouples and takes the form

$$
\partial_{z} u^{+}=\alpha u^{+}, \quad \partial_{z} u^{-}=-\alpha u^{-},
$$

where $\alpha$ is an operator involving a convolution and time derivatives. As shown for dispersive media modelled by (2.5), the decoupling of the first-order system does not guarantee that the pertinent function is forward or backward propagating.

In our approach, in the frequency domain, the homogeneity of the material implies that $\mathscr{F}=0$ and that $(4.18)$ reduces to

$$
w(z, t)=\frac{1}{2 \pi} \int_{-\infty}^{\infty} P(\omega) \exp \left[\int_{0}^{z} \Lambda(\zeta, \omega) d \zeta+i \omega t I\right] P^{-1}(\omega) w_{F}(0, \omega) d \omega .
$$


Express $w_{F}(0, \omega)$ as a linear combination of the two eigenvectors of $A, w_{F}(0, \omega)=$ $\beta_{1} p_{1}+\beta_{2} p_{2}$. Hence,

$$
\begin{aligned}
& P^{-1} w_{F}= {\left[\begin{array}{l}
\beta_{1} \\
\beta_{2}
\end{array}\right], } \\
& \begin{aligned}
w(z, t)=\frac{1}{2 \pi} \int_{-\infty}^{\infty}\left\{\beta_{1}(\omega) \exp \left[\lambda_{1}(\omega) z+i \omega t\right] p_{1}(\omega)\right. \\
\left.+\beta_{2}(\omega) \exp \left[\lambda_{2}(\omega) z+i \omega t\right] p_{2}(\omega)\right\} d \omega .
\end{aligned}
\end{aligned}
$$

Since $\lambda_{1}\left(\lambda_{2}\right)$ is associated with a backward- (forward-) propagating wave, it follows that $w(z, t)$ is the result of two waves in the time domain, namely

$$
\begin{aligned}
w(z, t) & =w^{f}(z, t)+w^{b}(z, t), \\
w^{f}(z, t) & =\frac{1}{2 \pi} \int_{-\infty}^{\infty}\left\{\beta_{2}(\omega) \exp \left[\lambda_{2}(\omega) z+i \omega t\right] p_{2}(\omega)\right\} d \omega, \\
w^{b}(z, t) & =\frac{1}{2 \pi} \int_{-\infty}^{\infty}\left\{\beta_{1}(\omega) \exp \left[\lambda_{1}(\omega) z+i \omega t\right] p_{1}(\omega)\right\} d \omega,
\end{aligned}
$$

$w^{f}$ being forward propagating and $w^{b}$ backward propagating.

ACKNOWLEDGMENTS. The research leading to this work was partially supported by the MIUR Research Project "mathematical models for materials science." The authors are grateful to the anonymous referee for bringing [4] to their attention.

\section{REFERENCES}

[1] I. Åberg, G. Kristensson, and D. J. N. Wall, Transient waves in nonstationary media, J. Math. Phys. 37 (1996), no. 5, 2229-2252.

[2] G. Caviglia and A. Morro, Reflection and transmission in pre-stressed stratified multilayers, Acta Mech. 153 (2002), no. 1-2, 113-126.

[3] M. Fabrizio and A. Morro, Mathematical Problems in Linear Viscoelasticity, SIAM Studies in Applied Mathematics, vol. 12, Society for Industrial and Applied Mathematics (SIAM), Pennsylvania, 1992.

[4] S. He, Factorization of a dissipative wave equation and the Green functions technique for axially symmetric fields in a stratified slab, J. Math. Phys. 33 (1992), no. 3, 953-966.

[5] S. He, S. Ström, and V. H. Weston, Time Domain Wave-Splittings and Inverse Problems, Oxford University Press, Oxford, 1998.

[6] D. Porter and D. S. G. Stirling, Integral Equations, Cambridge Texts in Applied Mathematics, Cambridge University Press, Cambridge, 1990.

G. CAVIGLIA: DEPARTMENT OF MATHEMATICS, DIMA, UNIVERSITY OF GENOA, ViA DODECANESO 35, 16146 GENOA, ITALY

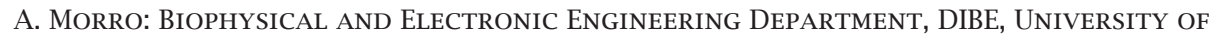
GenoA, Via Opera Pia 11A, 16145 Genoa, ItAly

E-mail address: morro@dibe.unige.it 


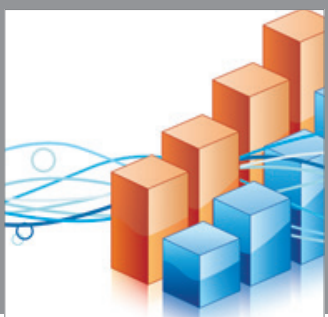

Advances in

Operations Research

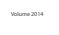

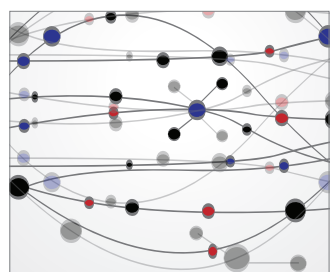

\section{The Scientific} World Journal
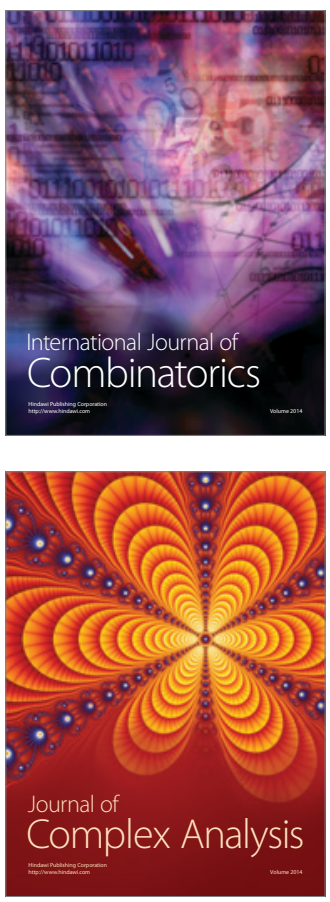

International Journal of

Mathematics and

Mathematical

Sciences
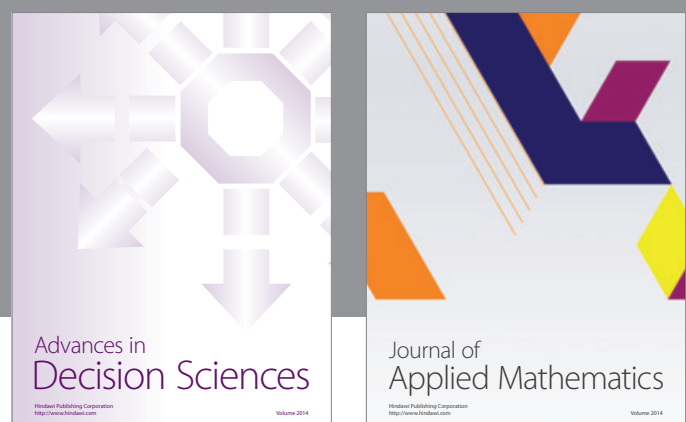

Journal of

Applied Mathematics
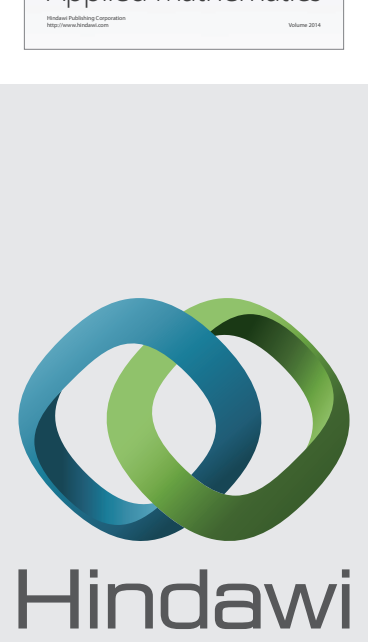

Submit your manuscripts at http://www.hindawi.com
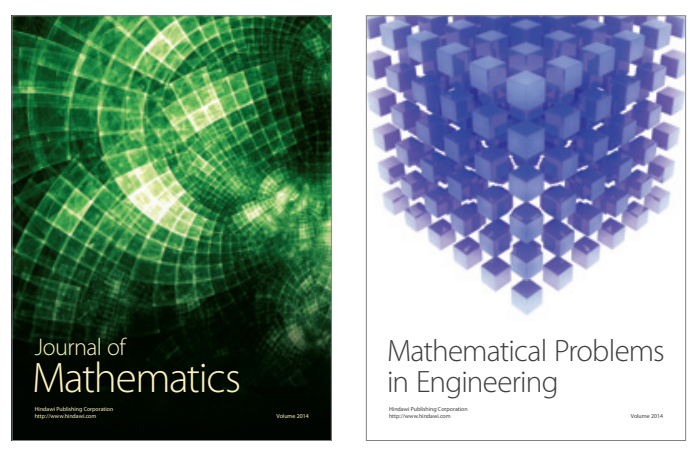

Mathematical Problems in Engineering
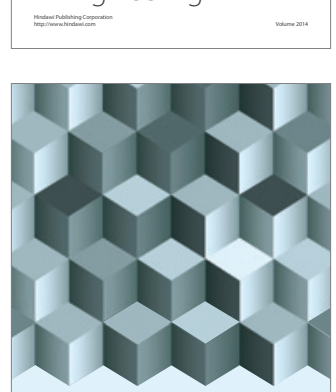

Journal of

Function Spaces
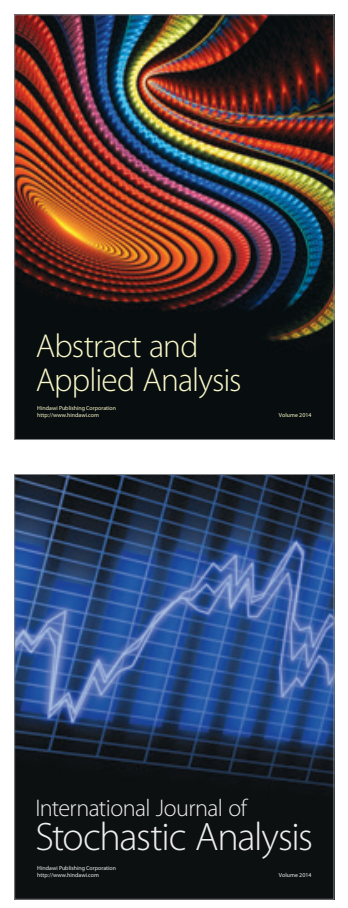

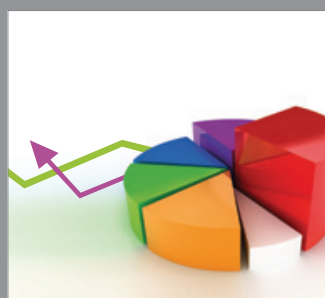

ournal of

Probability and Statistics

Promensencen
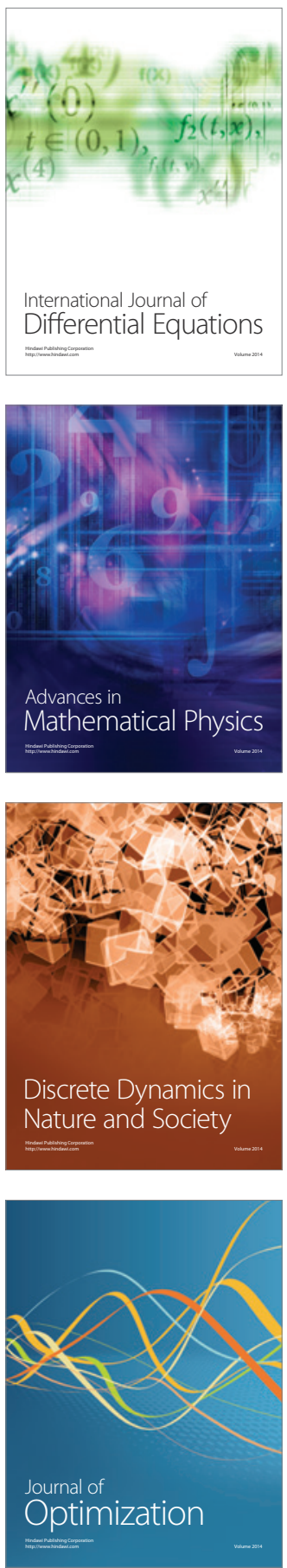